\title{
Exploration of Cache Behavior Using HPSS Per-File Transfer Logs
}

\author{
Harvard Holmes (hhholmes@lbl.gov) \\ Mass Storage Group \\ Nancy Meyer (Group Lead), \\ Matthew Andrews, Shreyas Cholia, Damian Hazen, \\ Wayne Hurlbert, Nancy Johnston \\ National Energy Research Scientific Computing Center \\ Lawrence Berkeley National Laboratory \\ Berkeley, CA 94720
}

November 2001

\begin{abstract}
We assembled 18 months of transfer logs from a production High Performance Storage System (HPSS) system at the National Energy Research Scientific Computing Center (NERSC) and analyzed them to assess workload behavior and gain some insight into which cache configurations would provide the best service to the users.

We found, as expected, that the workload is distributed over file size with a declining number of files as the files get larger, so the amount of space consumed per file size increment is roughly constant up to file sizes of 1 GB. Sixty one percent of file accesses were write accesses. There are a significant number of files written which are never read - backup files and similar files. For all sizes of files, access frequencies decline with the age of the files.

HPSS uses the cache as an I/O buffer for incoming data. At our installation the cache behavior is dominated by the write traffic. Cache lifetimes tend to scale linearly with the size of the cache and inversely with the amount of data flow.

This work was supported by the Director, Office of Science, Office of Advanced Scientific Computing Research, Mathematical, Information, and Computational Sciences Division, U.S. Department of Energy under Contract No. DE-AC03-76SF00098.
\end{abstract}




\section{Introduction}

HPSS was developed by a consortium of government, industrial, and educational sites [1] and is currently deployed at many large supercomputing sites. NERSC is a developer site within the consortium. HPSS provides a high performance data storage architecture based on the IEEE Mass Storage Reference Model [3, 4], which is designed to operate in a multiple-computer parallel configuration. The HPSS facility is a key component among the computational resources provided to NERSC users, responding to the increasingly data-intensive aspects of modern computational science.

NERSC has two HPSS systems ("Archive" and "HPSS"), both of which are accessible interactively from all NERSC supercomputers and auxiliary systems, as well as from offsite computers. Both systems are also accessible to batch jobs from all NERSC systems [2]. Of the two systems, user archive activity is encouraged on "Archive," and backup and other system activities are encouraged on "HPSS." Each system has an IBM AIX SP Silver node for file system management and other metadata operations, and 4 Winterhawk nodes for data movement. The systems had 3 TB (Archive) and 3.3 TB (HPSS) of disk cache at the time of this study. The systems share 60 StorageTek 9840 tape drives in 8 StorageTek tape libraries. Collectively, the systems store about 200 TB of data in about 12 million files, and perform about $900 \mathrm{~GB}$ of user I/O per day [5]

\section{NERSC 's HPSS Hardware Architecture}

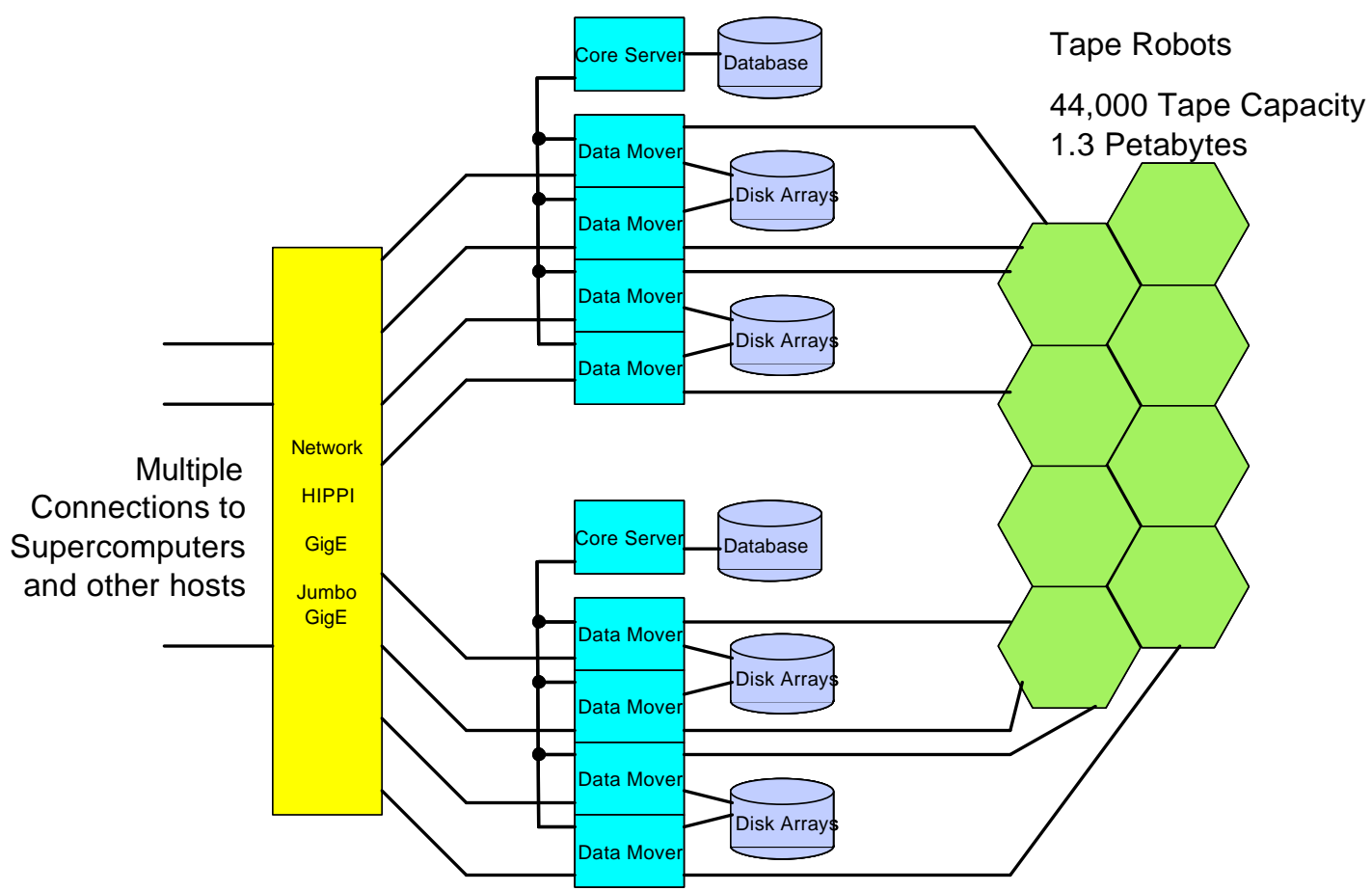

18 TB of temporary disk storage

Figure 1. NERSC's Hardware Architecture 


\section{Configuration Choices in HPSS}

Both systems are configured with multiple Classes of Service (COS), which segment the disk cache according to file sizes and other properties. Users may also specifically select a COS for special file usages, such as backups. Generally the systems have COSs for small (0 to $2 \mathrm{MB}$ ) files, medium (2 MB to $100 \mathrm{MB}$ ) files, and large (over $100 \mathrm{MB}$ ) files. Some sites also have categories of service similar to the above which provide multiple tape copies of files for offsite storage.

More detailed configuration choices in HPSS involve the allocation of disk resources to specific storage classes, the configuration of disk resources into allocation blocks, and the choice of purge and migration policies.

\section{Issues Involved in Selecting Storage Class Sizes and COS Size Limits}

- Minimize wasted space on disk and tape;

- Maximize writing efficiency to tape (taken as constant in this analysis);

- Minimize reading costs;

- Minimize repack costs (not addressed in this analysis).

\section{Data Collection}

The primary interfaces to our HPSS systems are PFTP and HSI. PFTP is the HPSS supplied parallel FTP client, and HSI is the Hierarchical Storage Interface developed by Mike Gleicher with funding from several of the development sites. Both of these interfaces create both detailed and transfer log files as text files. The detail logs record a great deal of internal operational data, while the transfer logs record one entry for each file transferred to or from a client. The transfer logs are saved and used for statistical summaries and to extract individual usage information. Each interface type creates its own distinct log file on the machine where it runs and the log files of different types and from the various machines must be combined later. The transfer logs were deemed most suitable for this investigation. Information included in the transfer logs includes the date and time of the transfer, the time taken for the transfer or the data rate, the host which was the client, the size and name of the file transferred, and the user who transferred the file (not necessarily the owner of the file). Unfortunately, the transfer logs do not include information about file deletions. The lack of file deletion information prevents the complete reconstruction of the state of the cache, but the overall growth of the system is not largely different from the growth indicated by the transfer logs, so we believe that our exploration of cache behavior is still relevant. It is also the case that many of the files which are deleted are backup files, which are not deleted until weeks or months after they are stored. These files have generally already been purged from the cache, so their lack of deletion information in the log files does not affect our analysis. 
Over time, the formats of the transfer records have had several changes and revisions. A PERL script was written to examine each record and deduce the format and rewrite selected information in a standard form, including:

- the date and time of the transfer,

- the size of the transfer,

- whether the transfer was a read or write, and

- the full path name of the transfer (occasionally only the name is available).

This standard form is then sorted by the full path name. Workload characterization is performed using this sorted file. Using the sorted file, we replaced file path names with sequence numbers to make the file smaller for subsequent cache simulations. However, we added a field for the file extension, in case we wished to categorize files by type of file in later analyses. This created a second standard form, similar to the first form, but with the added field of the file extension. This second form was then sorted by date and time and passed to the cache simulation routines.

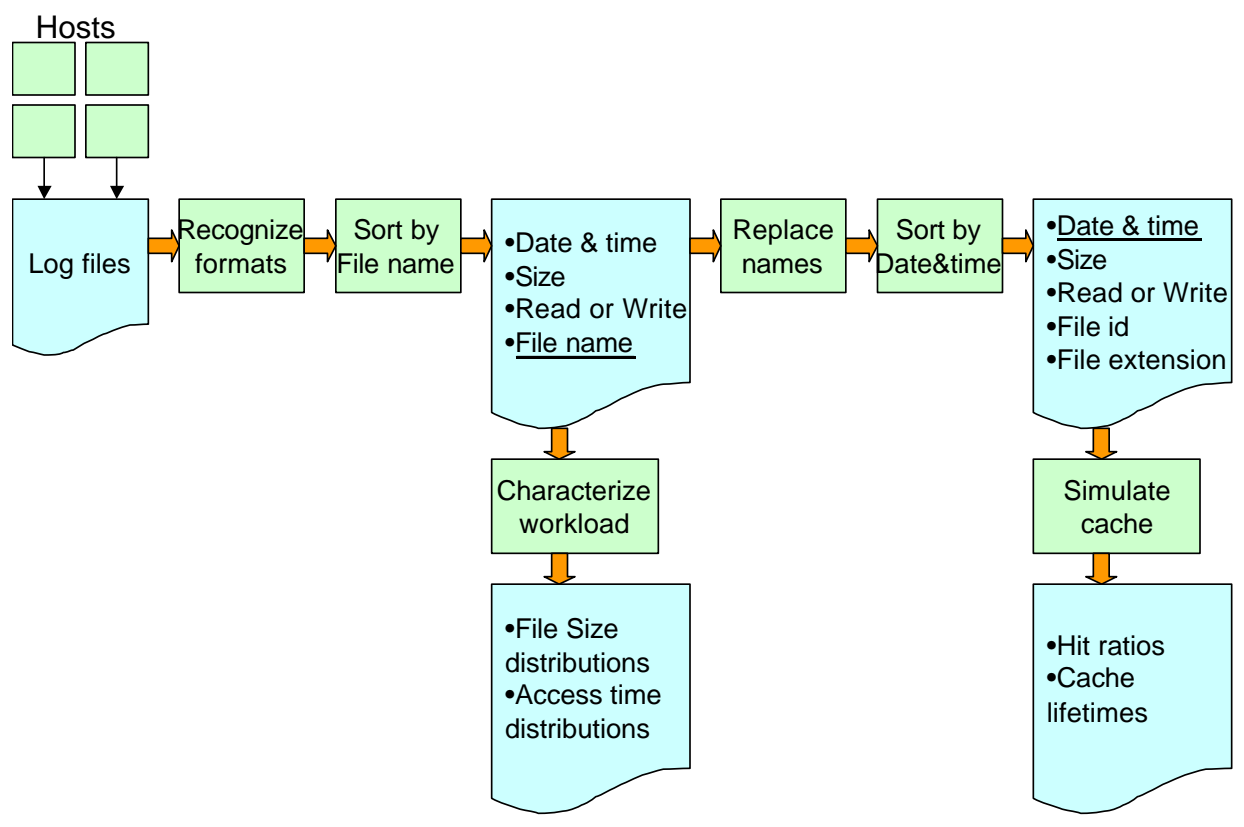

Figure 2. Data Processing Flow

\section{Workload Characterization}

During the time of the trace files, the total space utilized in both storage systems at NERSC grew from 111 TB at the end of 1999 to 213 TB in July of 2001 for a growth of 102 TB. Our logs show the total number of files written or overwritten as 178 TB. Subtracting this number from the growth yields the estimate that $76 \mathrm{~TB}$ of files were deleted or overwritten during the period.

\section{File Counts by Size Category}

Our first workload characteristic is simply the file counts and the amount of space transferred. The counts and amounts are listed for the totals and for the read subset. The 
"newfiles" are the number of unique file names in the log files. The analysis program does not have access to the existing directory of files, so a "newfile" can arise through writing a truly new file or through reading a file that has not been seen before by the analysis program. The graph of space density is obtained by dividing the GB in each category by the width of the category ("upto_GB" minus "from GB").

\begin{tabular}{|c|c|c|c|c|c|c|}
\hline \multicolumn{7}{|c|}{$\begin{array}{l}\# \text { "Archive" } \\
\# \text { Summary of io activity from transfer logs } \\
\#===================================\end{array}$} \\
\hline from_GB & upto_GB & io_GB & read_GB & io_cnt & read_cnt & newfiles \\
\hline 0.0000 & 0.0001 & 13.034 & 4.021 & 908629 & 235495 & 536329 \\
\hline 0.0001 & 0.0003 & 38.909 & 10.247 & 225874 & 55551 & 162341 \\
\hline 0.0003 & 0.0010 & 127.432 & 38.665 & 206953 & 62177 & 115188 \\
\hline 0.0010 & 0.0030 & 511.700 & 254.441 & 336471 & 181670 & 147465 \\
\hline 0.0030 & 0.0100 & 1616.117 & 435.771 & 254880 & 68606 & 191410 \\
\hline 0.0100 & 0.0320 & 2923.993 & 1086.314 & 147252 & 55298 & 94972 \\
\hline 0.0320 & 0.1000 & 10069.235 & 2951.907 & 183206 & 53459 & 96499 \\
\hline 0.1000 & 0.3200 & 27894.638 & 17094.732 & 80 & 89297 & 71808 \\
\hline 0.3 & 1. & 37166.863 & 185 & 61 & 39145 & 40023 \\
\hline 1.0000 & 3.0000 & 8193.525 & 1041.820 & 5639 & 598 & 4897 \\
\hline 3.0000 & 10.0000 & 10800.173 & 3344.556 & 2616 & 934 & 1625 \\
\hline 10.0000 & 32.0000 & 3381.402 & 108.978 & 161 & 6 & 143 \\
\hline 32.0000 & 100.0000 & 424.257 & 107.627 & 7 & 2 & \\
\hline 100.0000 & 320.0000 & 622.263 & 0.000 & 4 & 0 & \\
\hline 320.0000 & 1024.0000 & 0.000 & 0.000 & 0 & 0 & \\
\hline 0.0000 & 1024.0000 & 103783.542 & 041.732 & 2492933 & 842238 & 146270 \\
\hline
\end{tabular}

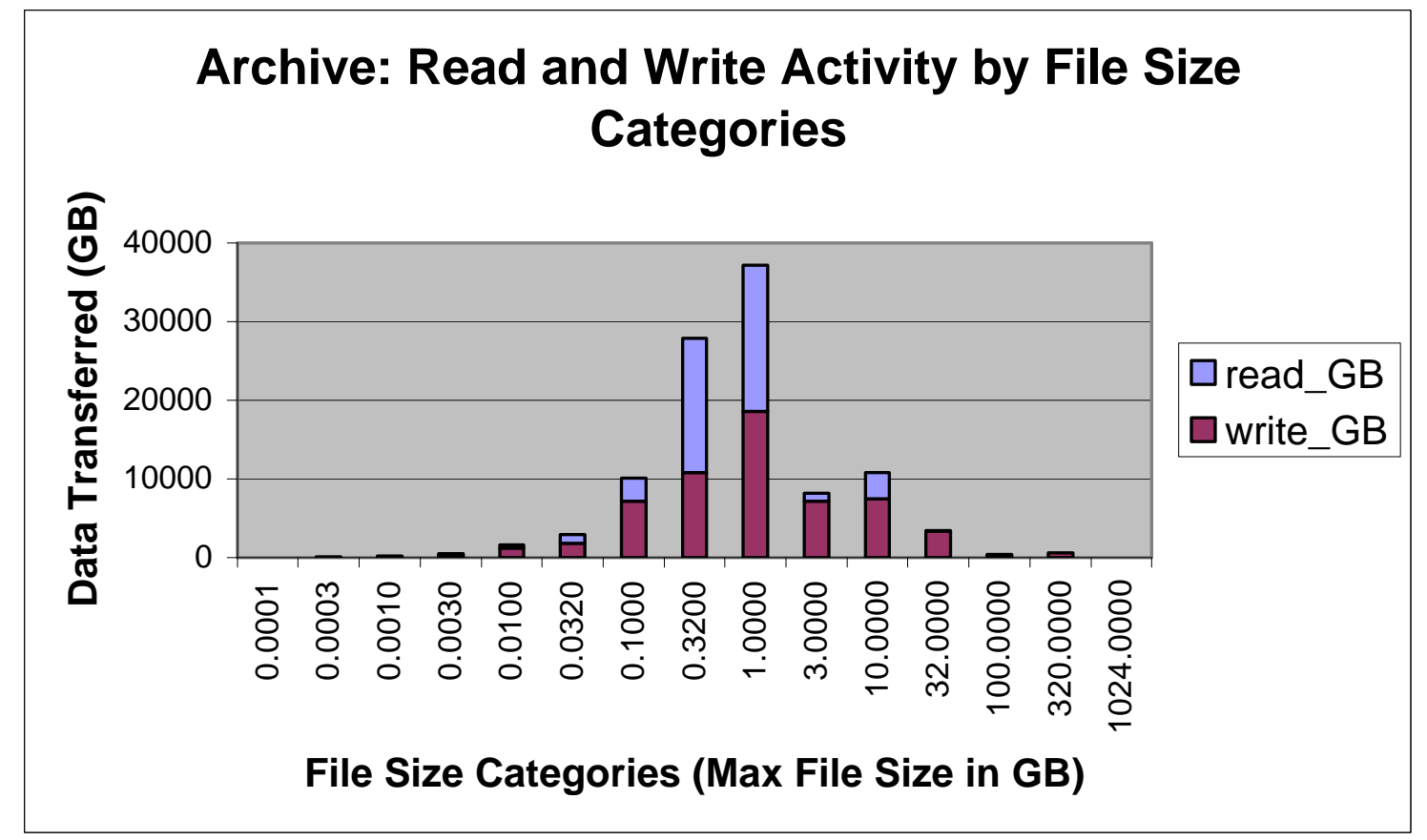

Figure 3. Read and Write Activity by File Size Categories for the Archive System 


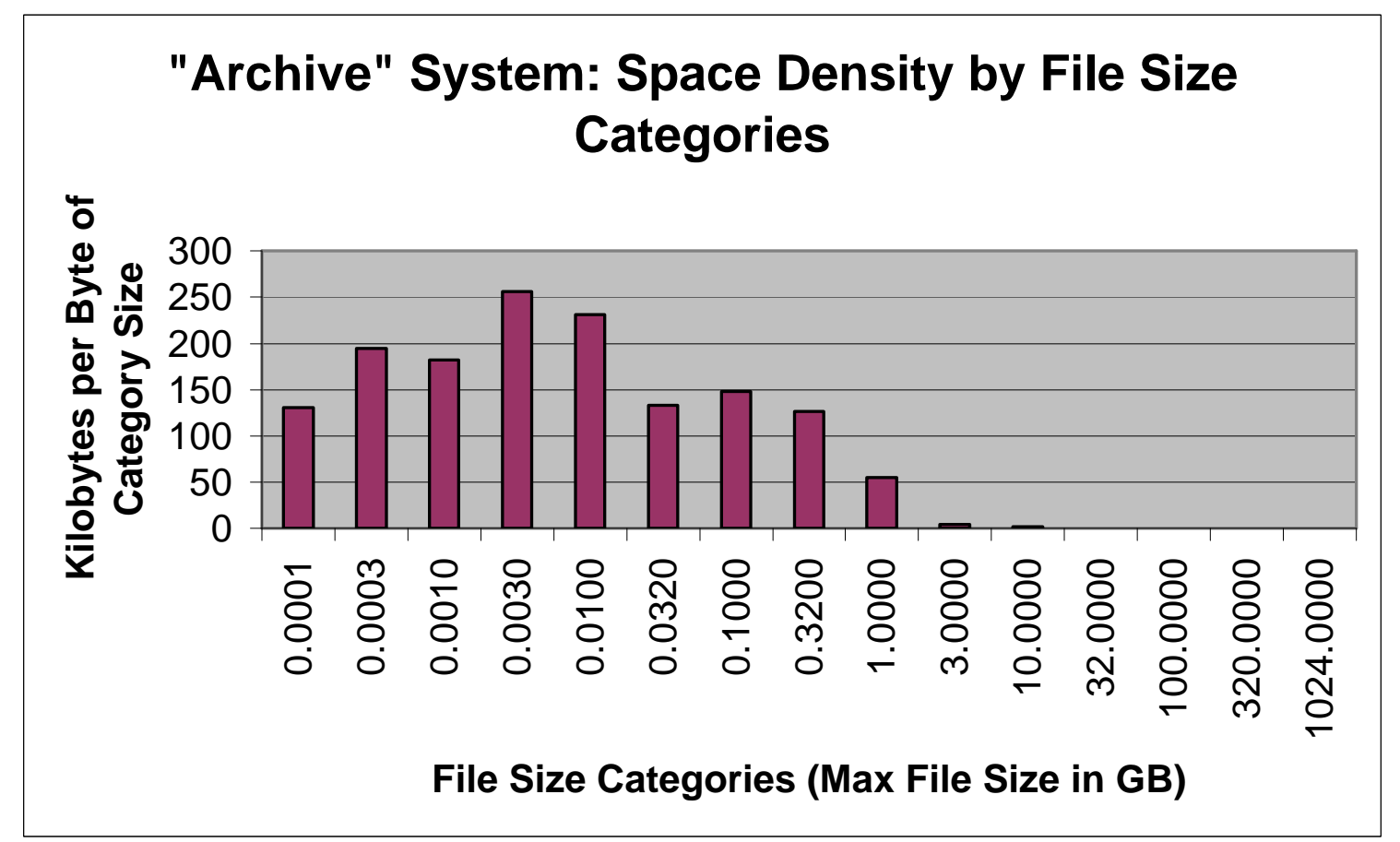

Figure 4. Space Density by File Size Categories for the Archive System

\begin{tabular}{|c|c|c|c|c|c|c|}
\hline \multicolumn{7}{|c|}{$\begin{array}{l}\text { \# "HPSS" } \\
\text { \# Summary of io activity from transfer logs } \\
\#======================================\end{array}$} \\
\hline from_GB & upto_GB & io_GB & read_GB & io_cnt & read_cnt & newfiles \\
\hline $0.0 \overline{0} 00$ & 0.0001 & $19 . \overline{9} 28$ & $3 . \overline{4} 65$ & $111 \overline{7} 654$ & 301651 & 600912 \\
\hline 0.0001 & 0.0003 & 35.571 & 11.495 & 186682 & 60385 & 137826 \\
\hline 0.0003 & 0.0010 & 208.649 & 53.763 & 353502 & 89654 & 235130 \\
\hline 0.0010 & 0.0030 & 1222.448 & 804.776 & 802683 & 553072 & 332135 \\
\hline 0.0030 & 0.0100 & 2745.203 & 1334.363 & 461461 & 223946 & 299510 \\
\hline 0.0100 & 0.0320 & 6122.866 & 1637.871 & 381031 & 103705 & 263734 \\
\hline 0.0320 & 0.1000 & 10148.328 & 2694.911 & 185211 & 42475 & 141886 \\
\hline 0.1000 & 0.3200 & 13990.182 & 4555.113 & 74218 & 22306 & 44653 \\
\hline 0.3200 & 1.0000 & 38262.774 & 7204.535 & 75058 & 13422 & 50445 \\
\hline 1.0000 & 3.0000 & 20061.262 & 1679.059 & 12212 & 1092 & 10249 \\
\hline 3.0000 & 10.0000 & 24711.444 & 4937.239 & 4755 & 921 & 3731 \\
\hline 10.0000 & 32.0000 & 23865.494 & 3402.440 & 1550 & 241 & 1254 \\
\hline 32.0000 & 100.0000 & 4943.268 & 0.000 & 118 & 0 & 117 \\
\hline 100.0000 & 320.0000 & 1644.745 & 0.000 & 11 & 0 & 11 \\
\hline 320.0000 & 1024.0000 & 0.000 & 0.000 & 0 & 0 & 0 \\
\hline 0.0000 & 1024.0000 & 147982.160 & 28319.031 & 3656146 & 1412870 & 2121593 \\
\hline
\end{tabular}




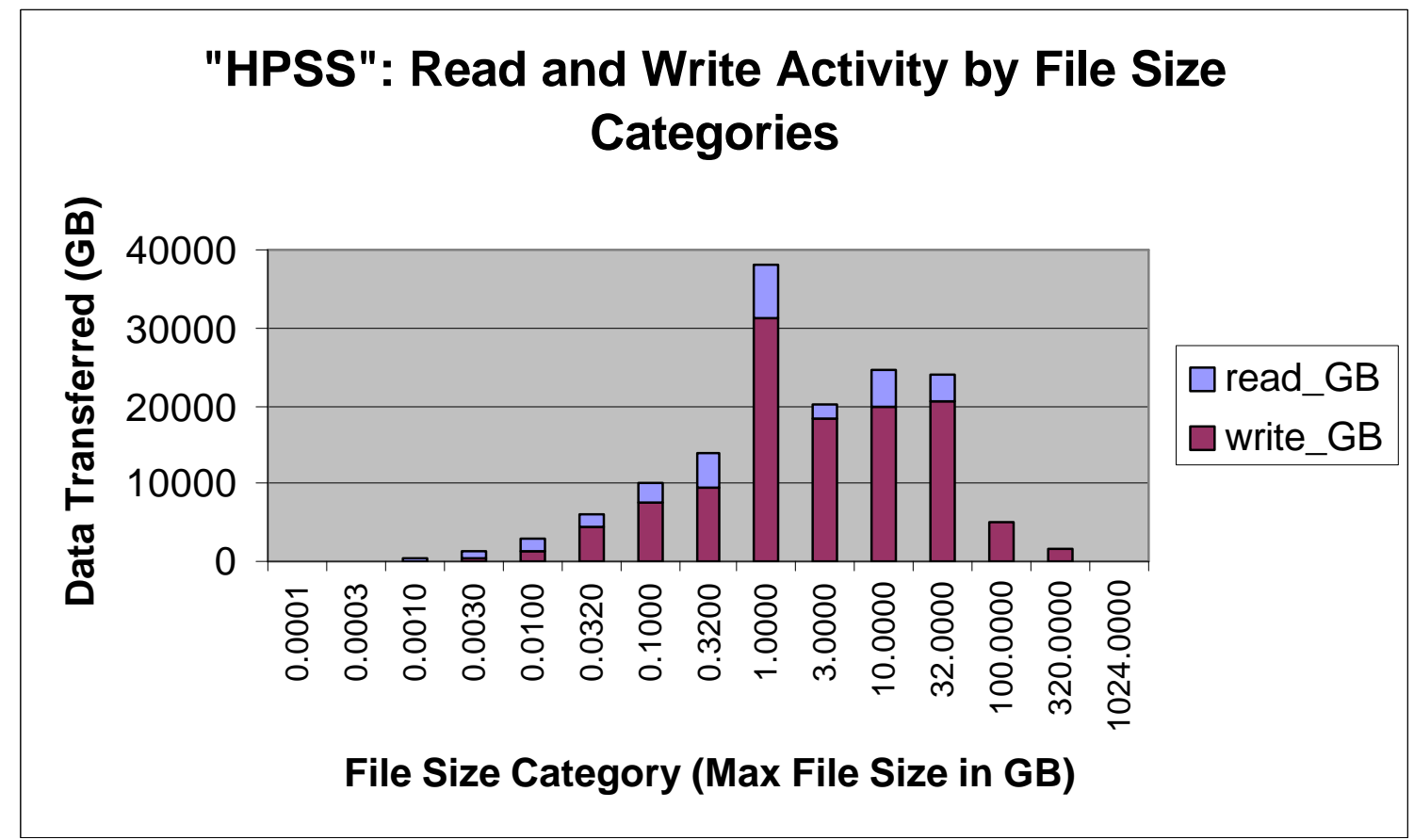

Figure 5. Read and Write Activity by File Size Categories for the "HPSS" System

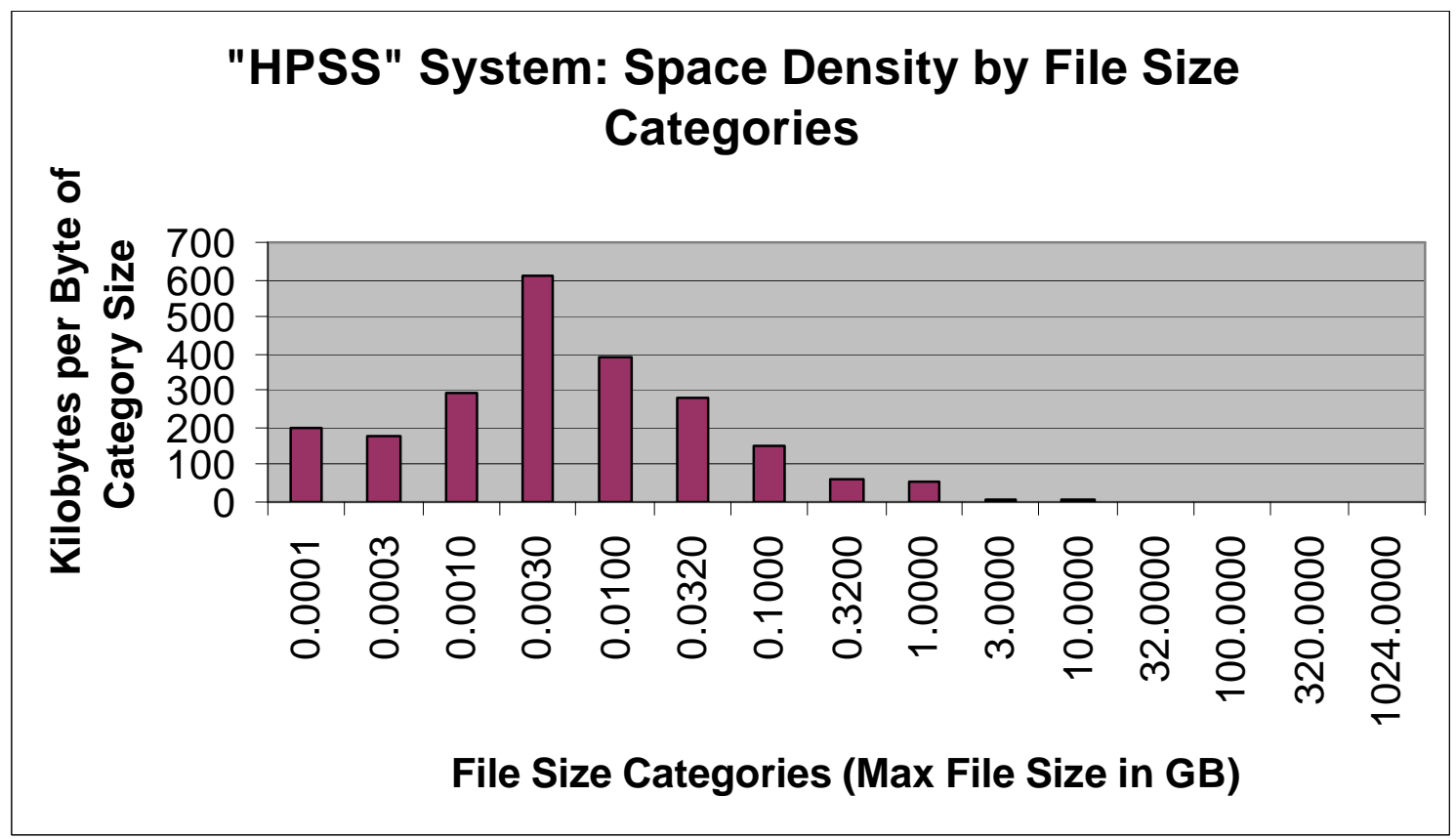

Figure 6. Space Density by File Size Categories for the "HPSS" System

\section{File Access Intervals}

A small extract of the access interval distribution is included below. The categories which are not shown have similar behavior - they all show the highest number of accesses on the same day as the previous access, with the rates declining steadily after 
that. The data for all files, and for aggregations into small, medium and large file sizes, is shown below for Archive. For "HPSS", we present only the graph of the access intervals for all files combined.

Note that there is some data censoring that we have not made any adjustments for. This data censoring reduces the frequencies of long access intervals and occurs because only the tail end of the analysis period can record long access intervals. For example, for a log file coverage of 600 days, only the last 300 days of the log can result in access intervals greater than 300 days, so the count of 300 day access intervals will be reduced by up to half from the rate that would have been observed with an infinite length log file coverage.

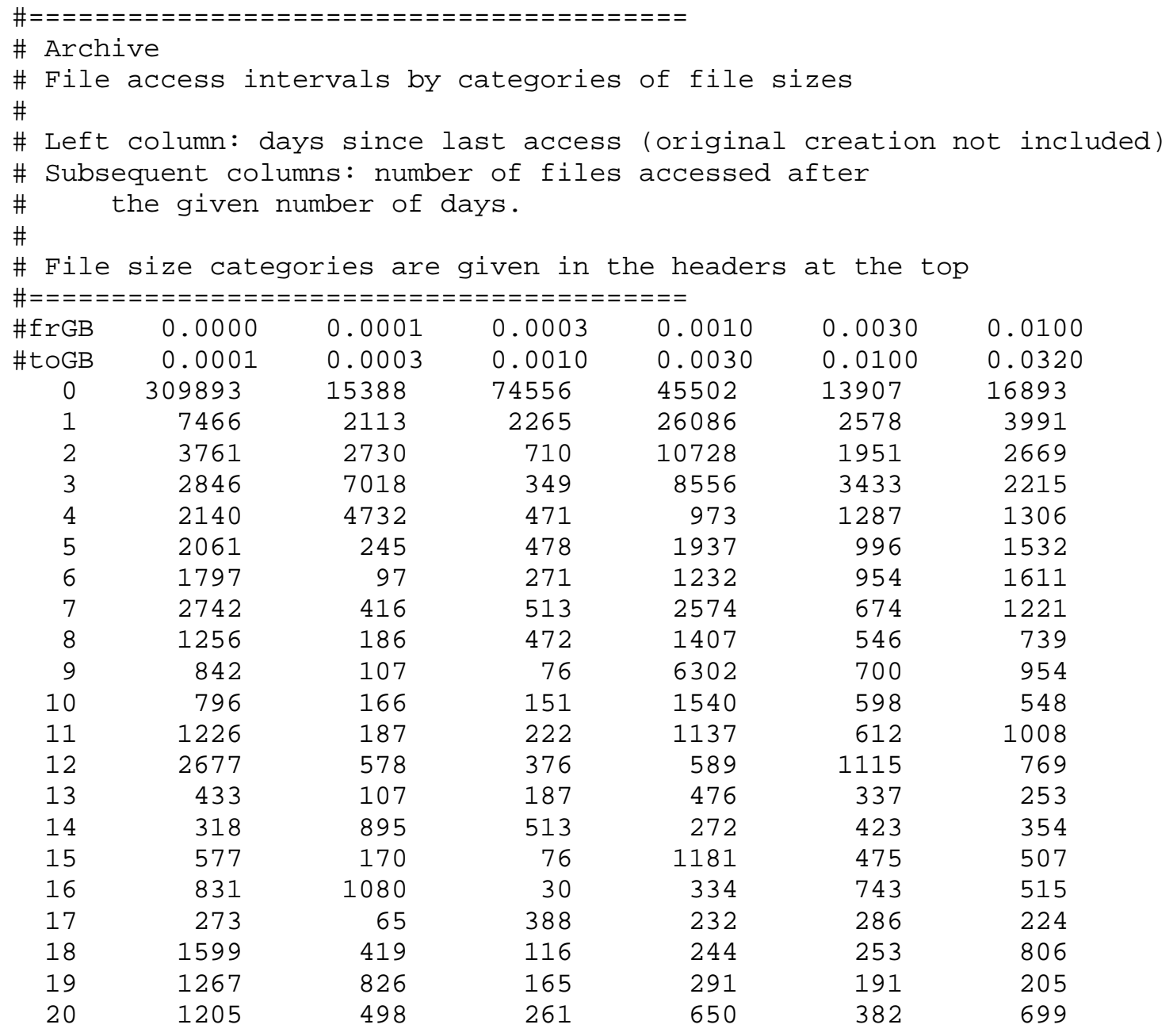




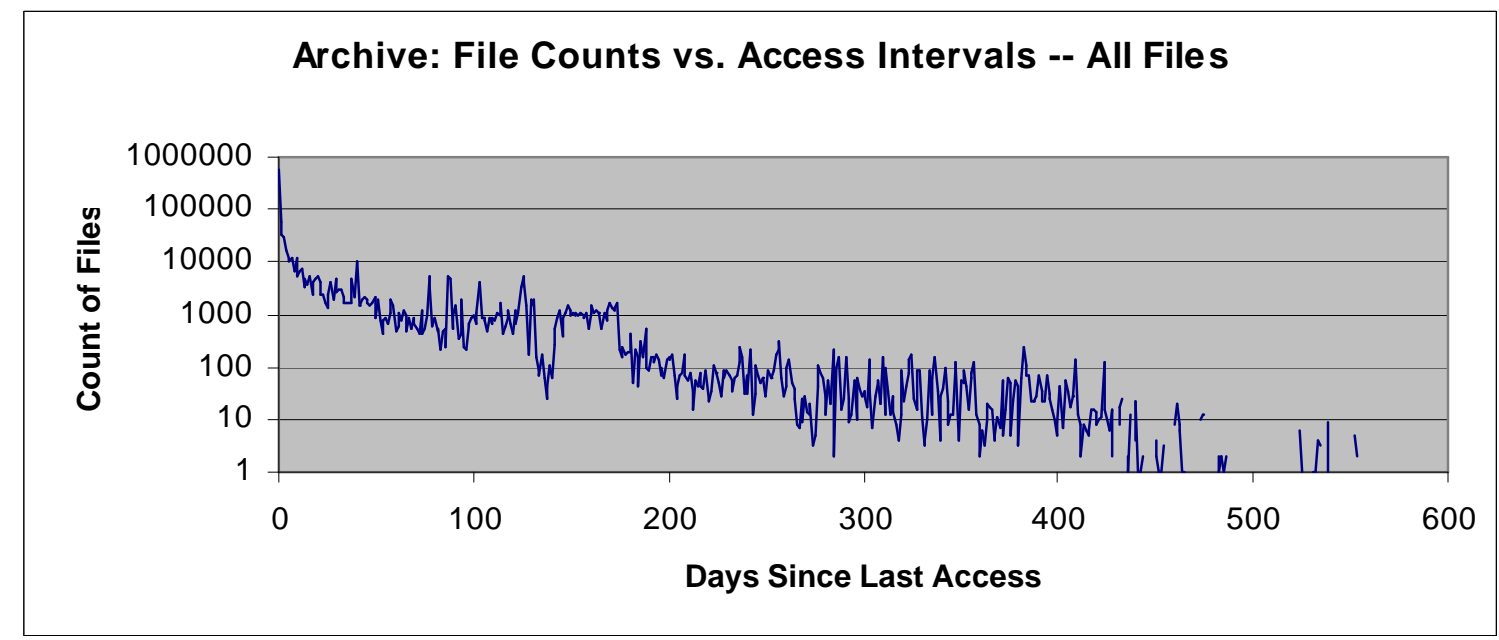

Figure 7. File Counts vs. Access Intervals for the Archive System - All Files

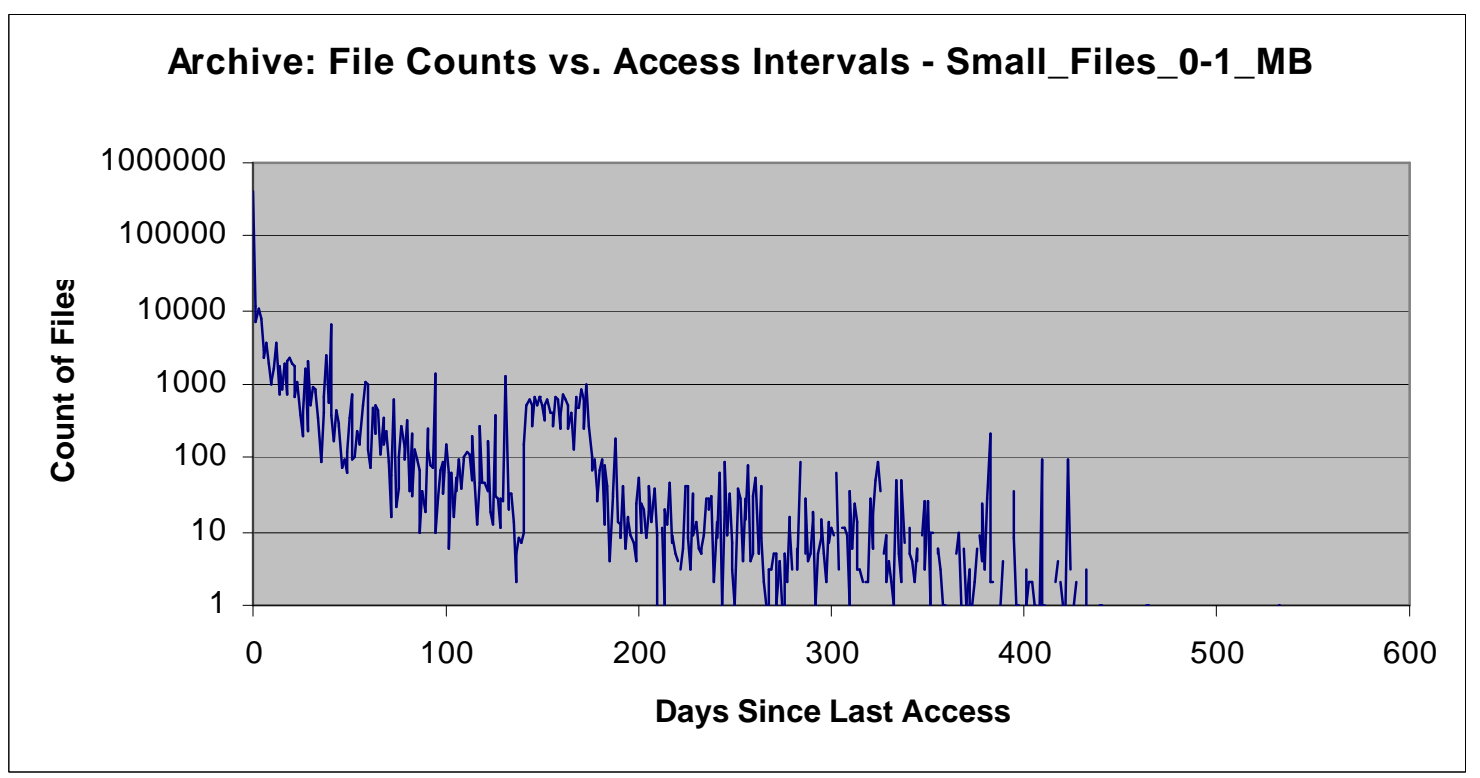

Figure 8. File Counts vs. Access Intervals for the Archive System - Small Files 


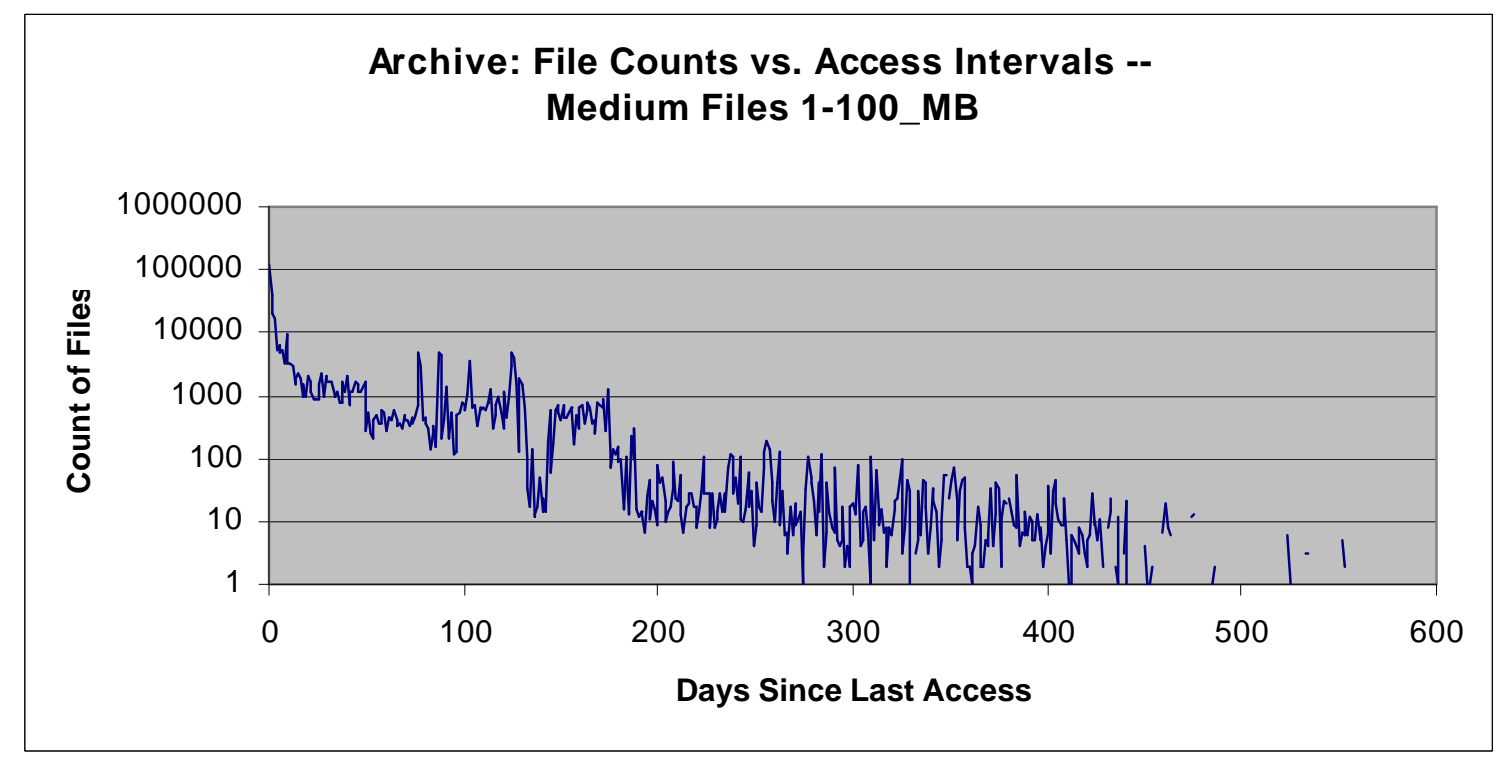

Figure 9. File Counts vs. Access Intervals for the Archive System - Medium Files

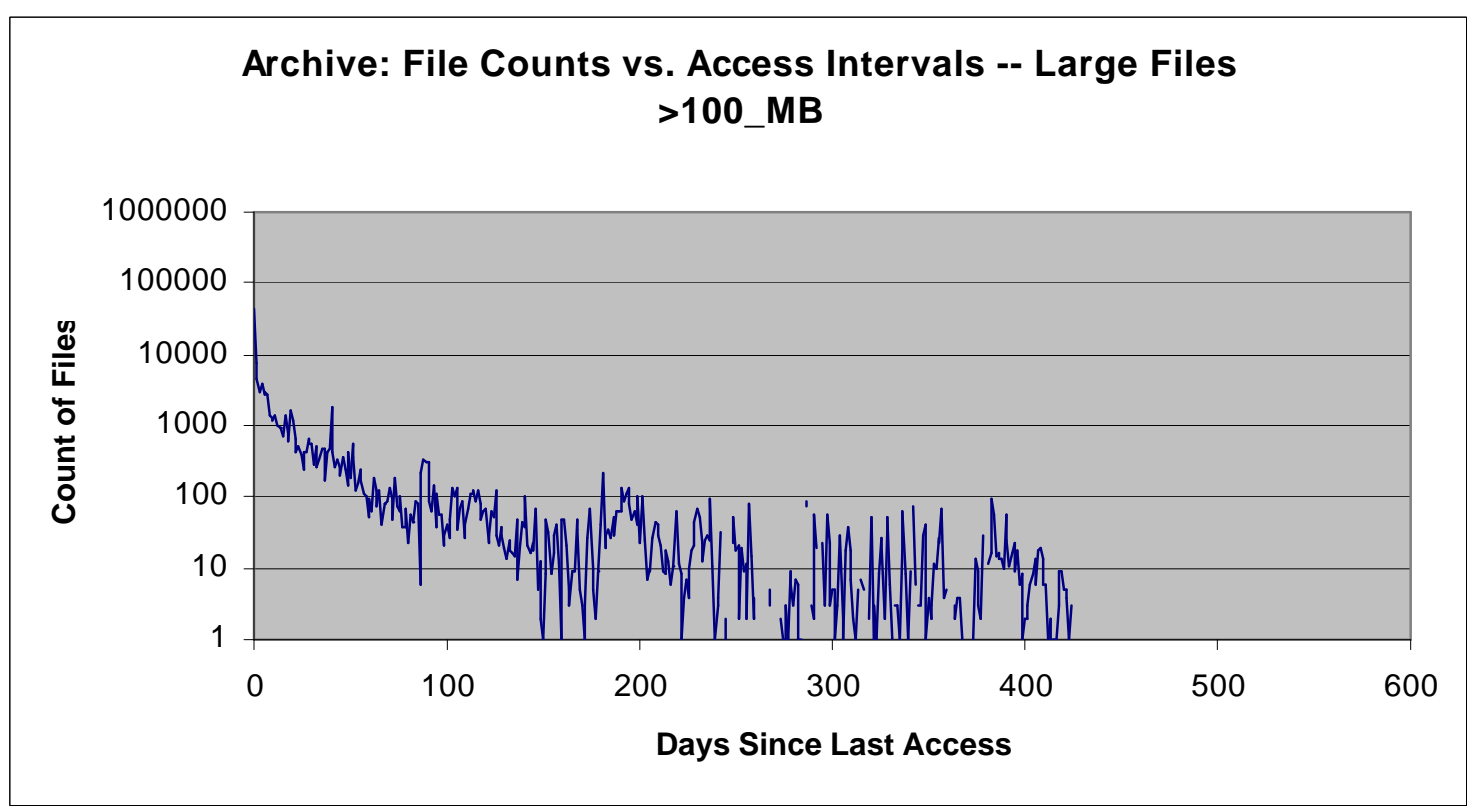

Figure 10. File Counts vs. Access Intervals for the Archive System - Large Files 


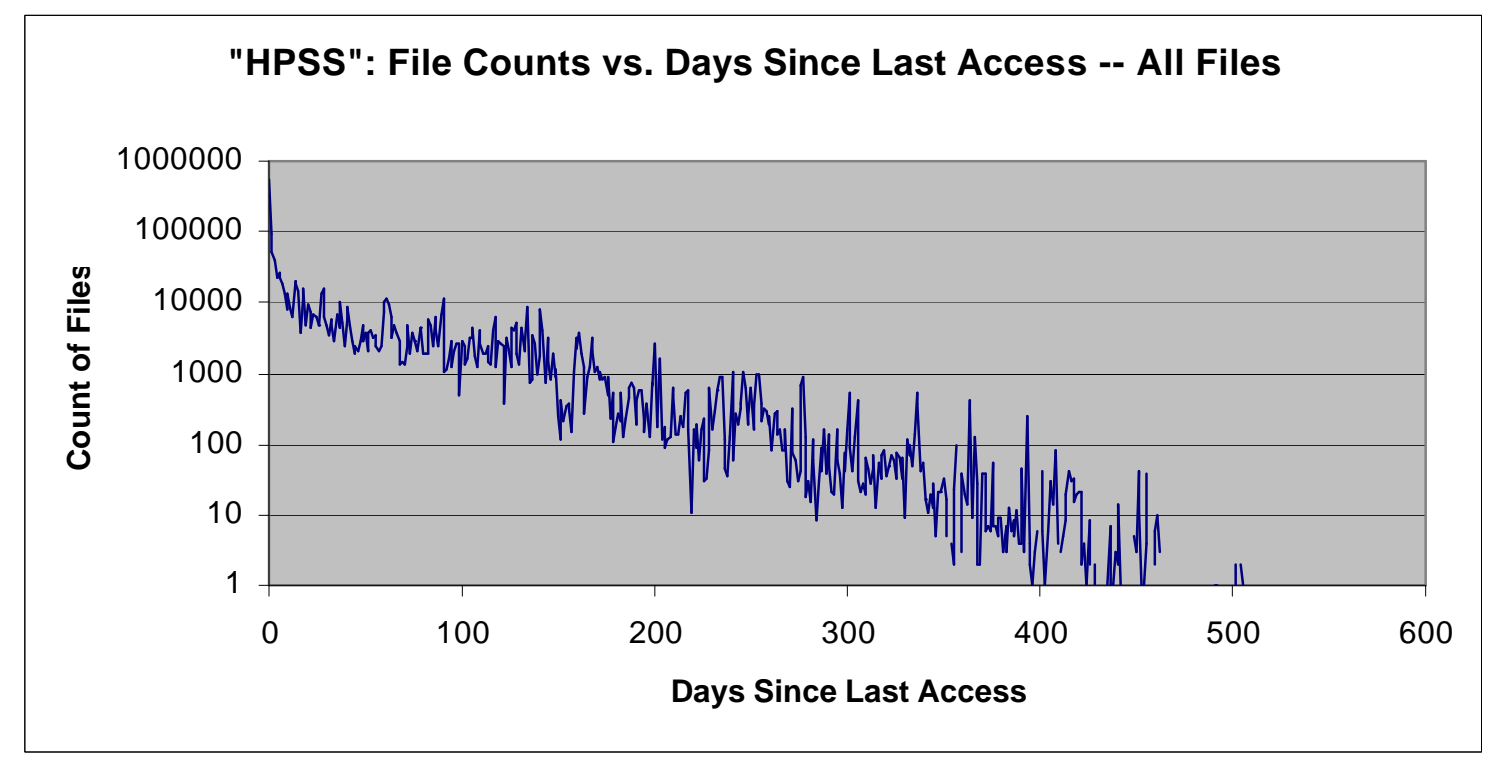

Figure 11. File Counts vs. Access Intervals for the "HPSS" System - All Files

\section{Cache Simulation}

To simulate the cache, a PERL script was written which simulates an LRU cache, with parameters for upper and lower bounds on file sizes, the cache size, and (for debugging) a limit on the number of records processed. This script will simulate 2 million operations in 5 to 10 minutes depending on the size of the cache (larger caches take longer). The simulations were run on an Intel laptop with $256 \mathrm{MB}$ of real memory and a $750 \mathrm{Mhz}$ CPU. The script records the number of files and the amount of data

- written into the cache (and thence written to tape),

- read from the cache, and

- read from tape (reads not in the cache).

Of course, the amount of data written is independent of cache size and is already known from the earlier file counts and data summaries. This figure serves as a convenient check. Another check is that the sum of data read from the cache plus data read from tape is a constant which does not depend on the size of the cache.

\section{Statistics Collected}

The cache statistics which are collected are fairly simple:

- the number of files which are read from the cache,

- the minimum and maximum cache residence times for all the files being simulated,

- an average cache residence time, computed using two different methods:

1. an actual average (later versions of the simulator only), and

2. from the data flow: cache size divided by the amount of data being put into the cache (writes to the cache plus reads from tape into the cache). 
A driver script was developed which ran the simulator with a systematic set of parameters to explore the parameter space of Class Of Service and cache configurations. To explore issues related to choices of file size categories, category boundaries were set up at 100 $\mathrm{KB}, 1 \mathrm{MB}, 10 \mathrm{MB}, 100 \mathrm{MB}, 1 \mathrm{~GB}, 10 \mathrm{~GB}, 100 \mathrm{~GB}$, and $1000 \mathrm{~GB}$. All possible combinations of category boundaries were simulated, e.g., 0 to $100 \mathrm{~KB}$, then 0 to $1 \mathrm{MB}$, and so forth. For each file size category, a simulation was run for cache sizes of $10 \mathrm{~GB}$, $100 \mathrm{~GB}, 1000 \mathrm{~GB}$, and $10 \mathrm{~TB}$. A sampling of this data is shown below. All file or data sizes are shown in GB. The headings are:
cos_min
lower limit of the file sizes simulated (GB)
cos_max
upper limit of the file sizes simulated (GB)
cache_sz
size of cache being simulated (GB)
tr_cnt
count of files simulated (meeting the size limits)
tp_w_dt
amount of data written to tape (GB)
tp_r_ct
count of tape reads required for data retrieval
tp_r_dt amount of data read from tape (GB)
ch_r_ct
count of files read from the cache
ch_r_dt
amount of data read from the cache (GB)
ch_rs_m
minimum cache residence time during the simulation (days) (1000000
or blank indicates the cache never filled up during the simulation)
ch_rs_X
maximum cache residence time during the simulation (days) ( 0 or
blank indicates the cache never filled up during the simulation)
ch_rs_av
average cache residence time (days), computed from data flows

Selected "HPSS" cache simulation statistics:

\begin{tabular}{|c|c|c|c|c|c|c|c|c|c|c|c|}
\hline cos_min & cos_max & cach_sz & tr_cnt & tp_w_dt & tp_r_ct & tp_r_dt & ch_r_ct & ch_r_dt & ch_rs_m & ch_rs_x & ch_rs_av \\
\hline 0 & 0.001 & 10 & 1657838 & 195.42 & 161413 & 30.07 & 290277 & 38.66 & 2 & 63 & 24.97 \\
\hline 0 & 0.001 & 100 & 1657838 & 195.42 & 97101 & 19.58 & 354589 & 49.15 & 297 & 377 & 261.86 \\
\hline 0 & 0.001 & 1000 & 1657838 & 195.42 & 94968 & 19.06 & 356722 & 49.68 & 1000000 & 0 & 2625.06 \\
\hline 0 & 0.001 & 10000 & 1657838 & 195.42 & 94968 & 19.06 & 356722 & 49.68 & 1000000 & & 26250.57 \\
\hline 0.001 & 0.1 & 10 & 1830386 & 13766.05 & 872879 & 5973.8 & 50319 & 498.98 & 0 & 9 & 0.29 \\
\hline 0.001 & 0.1 & 100 & 1830386 & 13766.05 & 778081 & 5294 & 145117 & 1178.82 & 0 & 14 & 2.95 \\
\hline 0.001 & 0.1 & 1000 & 1830386 & 13766.05 & 573106 & 3808.8 & 350092 & 2664.02 & 17 & 82 & 32.03 \\
\hline 0.001 & 0.1 & 10000 & 1830386 & 13766.05 & 249289 & 1797.7 & 673909 & 4675.1 & 303 & 440 & 361.74 \\
\hline 0.1 & 1000 & 1000 & 167922 & 105700.1 & 23167 & 10810 & 14815 & 10968.7 & 0 & 20 & 4.83 \\
\hline 0.1 & 1000 & 10000 & 167922 & 105700.1 & 12974 & 4937.6 & 25008 & 16841.4 & 31 & 122 & 50.8 \\
\hline
\end{tabular}

\section{Cache Lifetimes}

An illustrative way to view the data is to look at cache lifetimes as a function of data flow through the system. "Data flow" is the sum of data flowing into the cache from user writes to the storage system plus data read from tape (into the cache and then to the user). Note that both axes of these curves are logarithmic to cover the wide span of data values. The graphs generally show rather linear behavior over a wide range of values. 


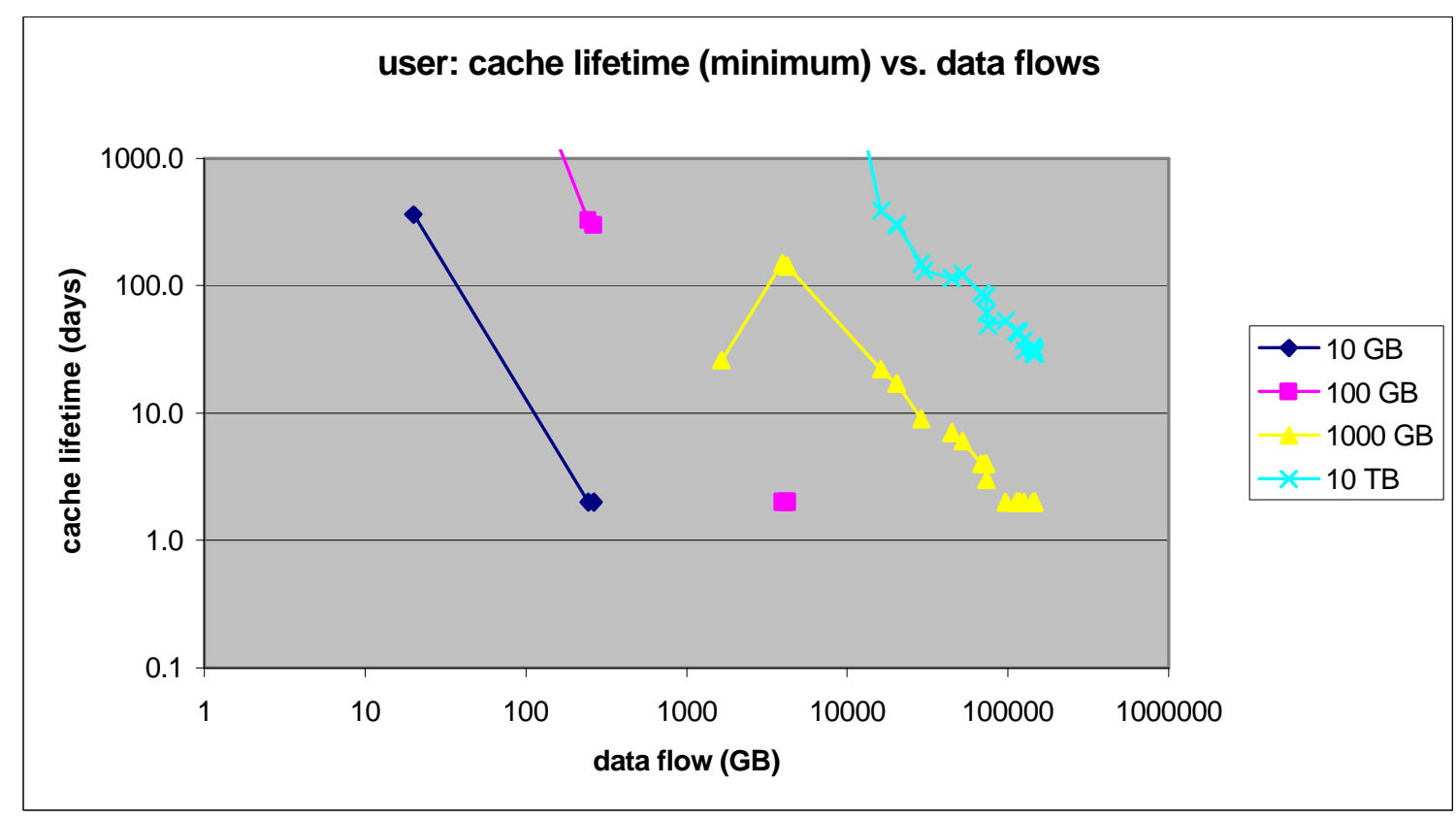

Figure 12. Cache Lifetime vs. Data Flows for the "HPSS" System - Minimum Lifetimes

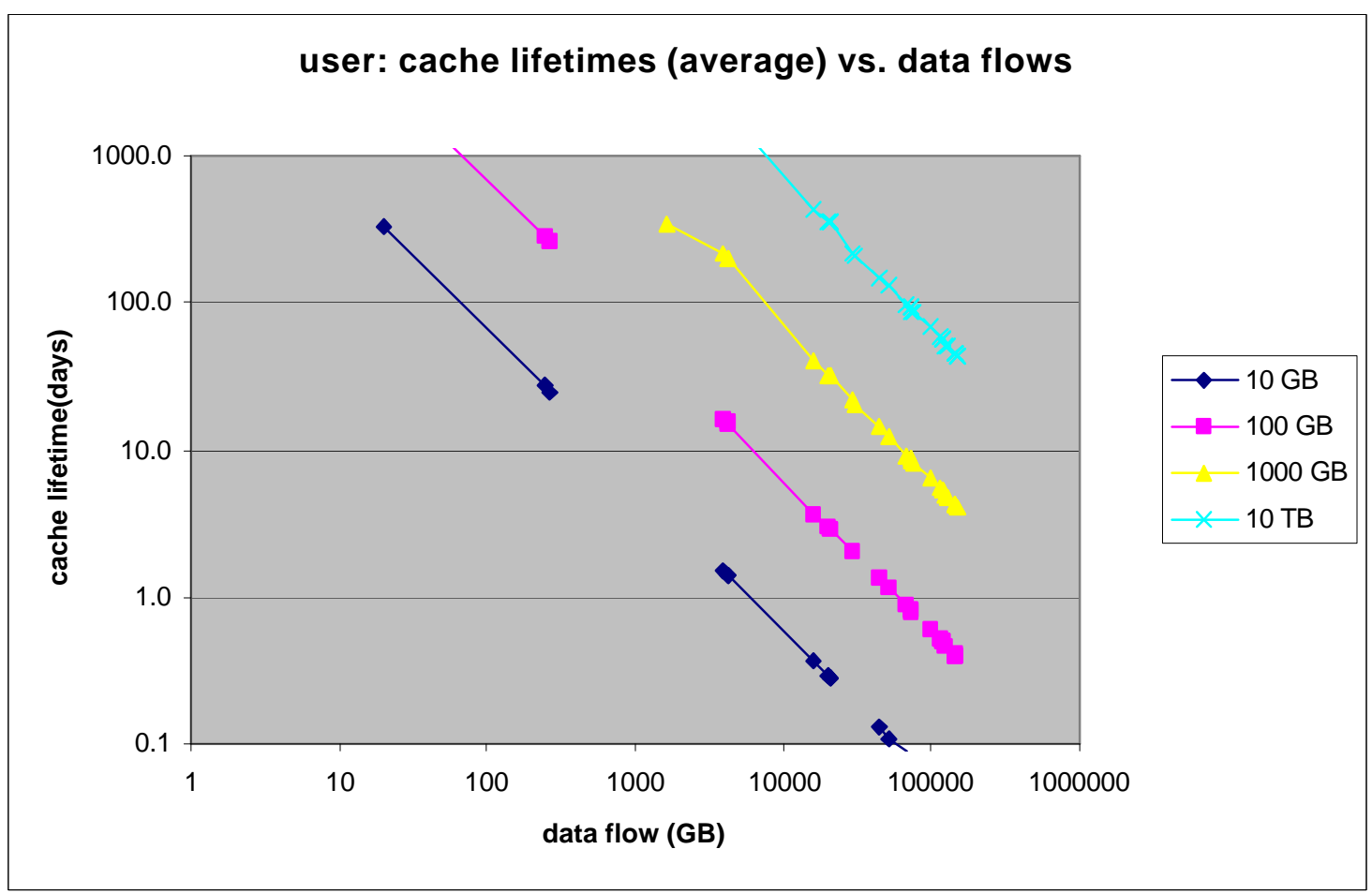

Figure 13. Cache Lifetime vs. Data Flows for the "HPSS" System - Average Lifetimes 


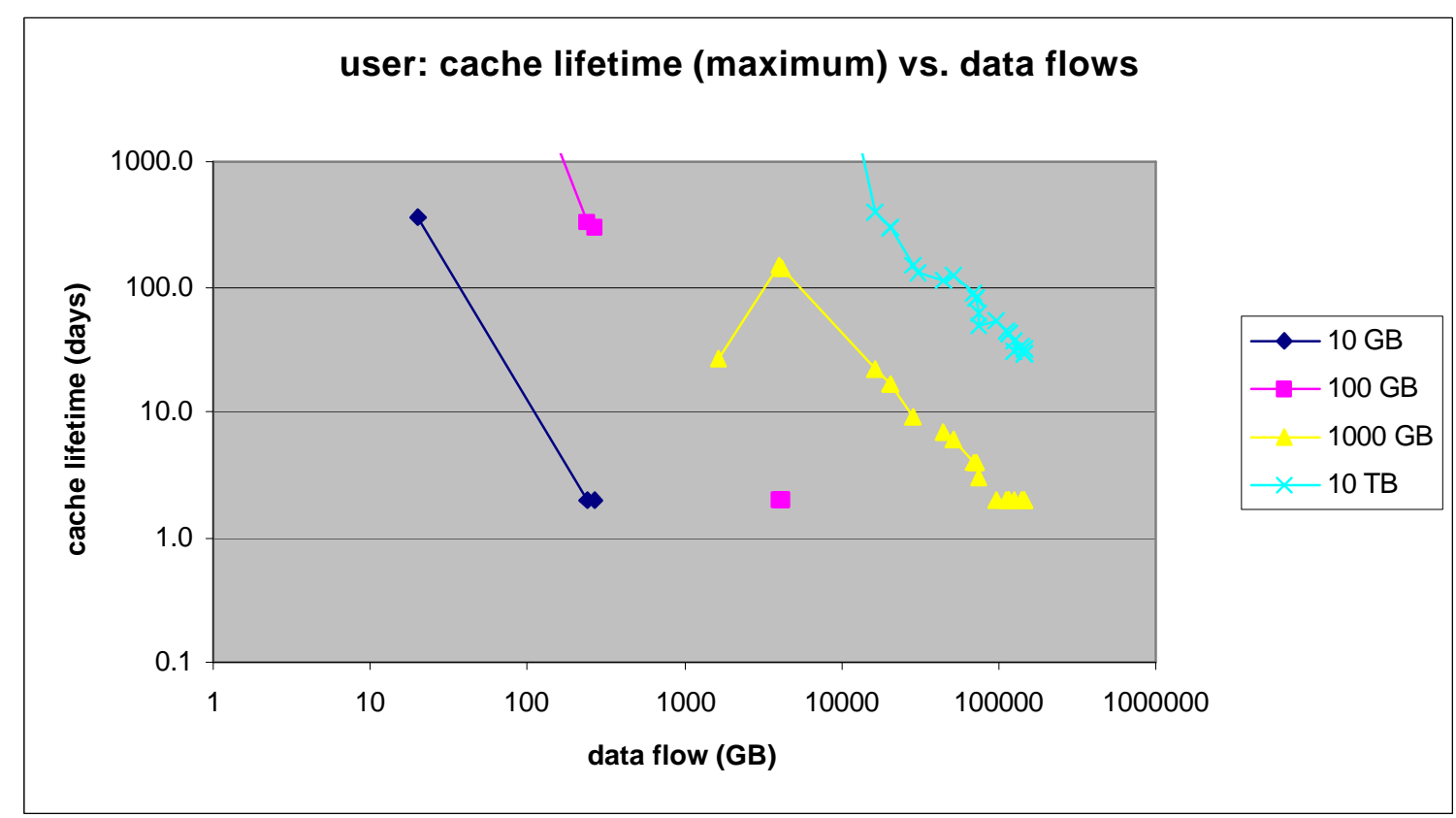

Figure 14. Cache Lifetime vs. Data Flows for the "HPSS" System - Maximum Lifetimes

\section{Sensitivity Analysis: Tape Mounts Required vs. Cache Increments}

We also ran the simulation for a few parameters with the size of the cache increased by $1 \%$ to see how this would affect the number of tape mounts required for reading data. Also shown is the increase to the next cache size in the standard set of parameters.

File sizes Cache increase Tape mount decrease Mount decrease/GB

0 to $1 \mathrm{MB}$ 10 to $10.1 \mathrm{~GB}$ 169 1690

0 to $1 \mathrm{MB}$

10 to $100 \mathrm{~GB}$

64312

715

1 to $100 \mathrm{MB}$

100 to $101 \mathrm{~GB}$

330

330

1 to $100 \mathrm{MB}$

100 to $1000 \mathrm{~GB}$

$100 \mathrm{MB}$ to $1 \mathrm{~TB}$

1000 to $1010 \mathrm{~GB}$

204975

1218

228

$100 \mathrm{MB}$ to $1 \mathrm{~TB}$

1000 to $10000 \mathrm{~GB}$

10193

121.8

\section{Sensitivity Analysis: Disk Allocation Size}

One of the configuration parameters in HPSS is the choice of the disk allocation size. To study the impact of this choice, simulation runs were made with several choice of disk allocation size. These runs were all made for a class of service from 0 to $1 \mathrm{MB}$ with 100 $\mathrm{GB}$ of cache. As can be seen from the graph, the number of tape reads required to satisfy the read requests is relatively constant up through $0.25 \mathrm{MB}$, then shows an increase by about $50 \%$ as the allocation size is increased to $1 \mathrm{MB}$. The number of requests satisfied from the cache shows the same decrease at $1 \mathrm{MB}$, but the change is smaller in proportion. The minimum cache lifetime is more interesting, showing a decrease starting about 16 $\mathrm{KB}$ and showing a steady drop from there all the way to $1 \mathrm{MB}$. All these results are consistent with the file inter reference intervals described earlier; since the number of references is concentrated in the short inter reference intervals, the cache effectiveness 
remains high even though the cache lifetime is declining. In the graph below, "tp_r_cnt" is the count of tape reads required to satisfy the read requests, "ch_r_ct" is the count of cache read requests required to satisfy the read requests, and "ch_rs_m" is the minimum cache residency time observed during the simulation using the specified disk allocation size.

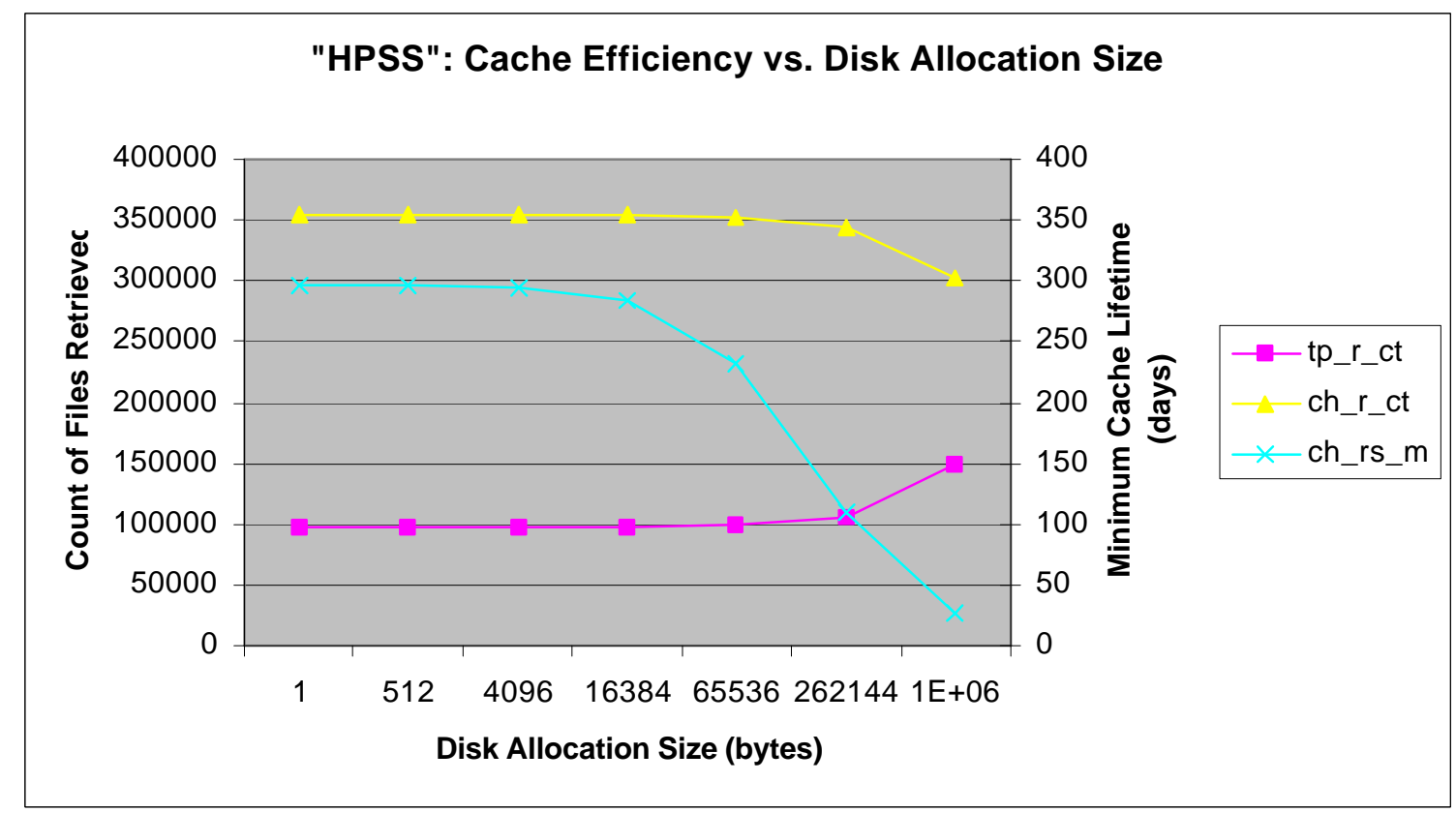

Figure 15. Cache Efficiency vs. Disk Allocation Size for the "HPSS" System

\section{Sensitivity Analysis: Purge Policy Choices}

Classes of Service use "hierarchies" to manage their storage, and hierarchies are composed of one or more "storage classes." Storage classes are more or less uniform types of devices, either disk or tape. Data movement between the storage classes is done according to "migration" and "purge" policies. Our main concern here is with purge policies, as these control the removal of files from the disk cache. HPSS in version 4.1 provides 3 choices of purge policy:

- Purge Record Creation Time (P) - this provides First In First Out behavior, suitable to meet certain fairness requirements,

- File Creation Time $(\mathrm{F})$ - to meet certain fairness requirements, and

- Last Data Access Time (L).

We made a few runs to compare the Purge Record Creation Time behavior with the Last Data Access Time behavior. It was surprising how little difference it made: 


\begin{tabular}{lrrrr} 
File sizes & Cache & Tape mounts(P) & Tape mounts(L) & Mount decrease \\
\hline 0 to $1 \mathrm{MB}$ & $10 \mathrm{~GB}$ & 162169 & 161424 & $745(0.5 \%)$ \\
0 to $1 \mathrm{MB}$ & $100 \mathrm{~GB}$ & 98590 & 97111 & $1479(1.5 \%)$ \\
1 to $100 \mathrm{MB}$ & $100 \mathrm{~GB}$ & 779417 & 778180 & $1237(0.2 \%)$ \\
1 to $100 \mathrm{MB}$ & $1000 \mathrm{~GB}$ & 579470 & 573159 & $6311(1.1 \%)$ \\
$100 \mathrm{MB}$ to $1 \mathrm{~TB}$ & $1000 \mathrm{~GB}$ & 23613 & 23188 & $425(1.8 \%)$ \\
$100 \mathrm{MB}$ to $1 \mathrm{~TB}$ & $10000 \mathrm{~GB}$ & 13413 & 12992 & $421(3.2 \%)$
\end{tabular}

\section{Observations}

Our observations from this simulation work is that our HPSS systems are rather dominated by the incoming write traffic and this tends to wash out nuances of behavior that might otherwise be observed. In particular, the write traffic tends to flush the cache on a very regular bases, producing two main effects:

1. The cache will never achieve near $100 \%$ coverage so the finer details of cache operation are mainly irrelevant, and

2. The constant flow of data through the cache makes it's operation rather linear and fairly easy to predict. This is particularly true for cache residence times.

We were surprised by the lack of affects in some of our sensitivity analyses. The sensitivities to disk allocation sizes and to the purge algorithm choice were less than we had anticipated.

On the other hand, the sensitivity of tape mount requests to cache size could provide a convenient mechanism to optimize the allocation of disk resources to caches for the various classes of service.

\section{Recommendations}

Several problems face the staff who are designing and installing HPSS. It is difficult to estimate the workload until the system has been in operation for some time, as the very existence of the system is likely to cause workload patterns to change from what they were when estimates of system load were initially made.

Most system administrators will be concerned with choosing appropriate classes of service and allocating disk resources among the classes of service to provide the best service to the users. The type of simulation technique described here can be used to explore options, provided the workload is similar in character. Our results suggest that the administrators need not worry too much about disk allocation sizes or purge policy choices. This type of simulation could also be used to make economic tradeoffs between disk and tape hardware, but we feel that a performance based approach (to provide users with good service) is more useful than an economic approach, and we have not applied such an economic approach to our own systems.

The file access interval data from the workload analysis is probably representative of a variety of systems and could be used to generate a synthetic workload for simulation runs 
such as we have done with real data. This would allow sites to explore options before installation, when insights are most needed and useful.

\section{Future Work}

An easy extension of this work is to sent the results of the simulation runs into an optimizer which would generate optimal disk cache sizes for a specific choice of file sizes in the Classes of Service. A further extension would undertake the automatic selection of file size boundaries for classes of service.

To accomplish these optimizations, it would be useful to have analytic expressions which would characterize the relevant parameters across the needed range of input parameter spaces.

Caching strategies which explicitly account for the heavy write traffic should be reviewed.

\section{Conclusions}

Our work has uncovered few surprises, but it has served to confirm our expectations. In the areas of purge policy choices and disk allocation sizes, we were somewhat surprised by the lack of sensitivity to these choices.

We have definitely gained a better insight into effective cache allocation and utilization.

The importance of write traffic on cache performance suggests that more work on cache algorithms that explicitly recognize write traffic might be well rewarded.

\section{Acknowledgement}

This work was supported by the Director, Office of Science, Office of Advanced

Scientific Computing Research, Mathematical, Information, and Computational Sciences Division, U.S. Department of Energy under Contract No. DE-AC03-76SF00098.

\section{References}

1. http://www.sdsc.edu/hpss/

2. $\quad$ http://hpcf.nersc.gov/storage/hpss/

3. R. A. Coyne and H. Hulen, "An Introduction to the Mass Storage Reference Model, Version 5," Proc. $12^{\text {th }}$ IEEE Symp. Mass Storage, Monterey, CA, IEEE Computer Society Press, April 1993.

4. IEEE Storage Systems Standards Working Group public documents, http://www.ssswg.org/public_documents/MSSRM/V5toc.html

5. $\quad$ http://hpcf.nersc.gov/storage/hpss/stats/monthly.html 


\section{Disclaimer}

This document was prepared as an account of work sponsored by the United States Government. While this document is believed to contain correct information, neither the United States Government nor any agency thereof, nor The Regents of the University of California, nor any of their employees, makes any warranty, express or implied, or assumes any legal responsibility for the accuracy, completeness, or usefulness of any information, apparatus, product, or process disclosed, or represents that its use would not infringe privately owned rights. Reference herein to any specific commercial product, process, or service by its trade name, trademark, manufacturer, or otherwise, does not necessarily constitute or imply its endorsement, recommendation, or favoring by the United States Government or any agency thereof, or The Regents of the University of California. The views and opinions of authors expressed herein do not necessarily state or reflect those of the United States Government or any agency thereof, or The Regents of the University of California.

Ernest Orlando Lawrence Berkeley National Laboratory is an equal opportunity employer. 